\title{
A Further Correspondence Property of $M$ Fractions
}

\author{
By J. H. McCabe
}

Abstract. It is shown that when all the coefficients, after the first, of one of the two corresponding series of an $M$ fraction are zero then the $M$ fraction has a symmetrical form.

Given a power series expansion of the form

$$
c_{0}+c_{1} z+c_{2} z^{2}+\cdots+c_{r} z^{r}+\cdots, \quad c_{0} \neq 0,
$$

and another of the form

$$
\frac{b_{1}}{z}+\frac{b_{2}}{z^{2}}+\frac{b_{3}}{z^{3}}+\cdots+\frac{b_{r}}{z^{r}}+\cdots, \quad b_{1} \neq 0,
$$

we can formally construct, by a variety of methods, the continued fraction

$$
\frac{c_{0}}{1+d_{1} z}+\frac{n_{2} z}{1+d_{2} z}+\frac{n_{3} z}{1+d_{3} z}+\cdots+\frac{n_{r} z}{1+d_{r} z}+\cdots,
$$

whose convergents will 'fit' $n$ terms of each series when expanded accordingly. That is, denoting the $n$th convergent by $A_{n}(z) / B_{n}(z)$ where clearly $A_{n}(z)$ and $B_{n}(z)$ are polynomials of degree $(n-1)$ and $n$, respectively, then

$$
\begin{aligned}
\frac{A_{n}(z)}{B_{n}(z)} & =c_{0}+c_{1} z+\cdots+c_{n-1} z^{n-1}+\text { higher order terms } \\
& =\frac{b_{1}}{z}+\frac{b_{2}}{z^{2}}+\cdots+\frac{b_{n}}{z^{n}}+\text { higher order terms. }
\end{aligned}
$$

One of the methods for obtaining the coefficients of the continued fraction from those of the two series is the q.d algorithm. Specifically, we use the recurrence relations

$$
n_{i+1}^{(k)}+d_{i}^{(k)}=n_{i}^{(k+1)}+d_{i}^{(k+1)}, \quad n_{i+1}^{(k)} * d_{i+1}^{(k+1)}=n_{i+1}^{(k+1)} * d_{i}^{(k)}
$$

for $k=0, \pm 1, \pm 2, \ldots$ and $i=0,1,2, \ldots$, with the starting values given by

$$
\begin{aligned}
d_{1}^{(k)} & =-c_{k} / c_{k-1}, & & k>0 \\
& =c_{0} / b_{1}, & & k=0 \\
& =-b_{-k} / b_{1-k}, & & k<0
\end{aligned}
$$

and

Received November 7, 1977.

AMS (MOS) subject classifications (1970). Primary 41 A20, 30A22.

Key words and phrases. Continued fractions. 


$$
n_{1}^{(k)}=0 \quad \text { for all values of } k \text {. }
$$

The coefficients of the continued fraction (3) are then those with $k=0$. The remaining coefficients generated are also those of continued fractions which correspond to the two series, but not equally to each series as (3) does. The continued fraction (3) is an $M$ fraction for the series (1) and (2). For full details of $M$ fractions, their correspondence properties and their relationship with Padé approximations see McCabe [2] and McCabe and Murphy [3].

When the two series represent some function in some region of the complex plane (one of the series may be asymptotic), the continued fraction has in many known cases shown fast convergence for both small and large values of $z$ in this region. See McCabe and Murphy [3] for some examples and also for some remarks concerning truncation errors. Sometimes only one or two terms of one series, usually that of the form (2), are available and these cases can also be dealt with. See McCabe [2]. In [5] Jones considers continued fractions of the form of $M$ fractions, referring to them as general $T$ fractions because of their similarity with the $T$ fractions of $\mathrm{W}$. J. Thron, and also relates them to two-point Padé approximants.

In this paper we show that in the special case when $b_{j}=0$ for $j>1$ then the $M$ fraction (3) has the symmetrical form

$$
\frac{c_{0}}{1+d_{1} z}+\frac{n_{2} z}{1-n_{2} z}+\frac{n_{3} z}{1-n_{3} z}+\cdots+\frac{n_{r} z}{1-n_{r} z}+\cdots+\cdots
$$

Thus, whatever method is used to generate the $n_{k}$ so that the fraction corresponds to the series (1), the denominator coefficients are simultaneously determined. As in the general case, $d_{1}=c_{0} / b_{1}$.

The result is established by making use of the following three properties of the convergents of (5), each of which is easily proved by induction:

1. $A_{n}(z)=A_{n-1}(z)+(-)^{n-1} n_{2} n_{3} \cdots n_{n} z^{n-1}$,

2. $B_{n}(z)=B_{n-1}(z)+(-)^{n-1} d_{1} n_{2} \cdots n_{n} z^{n}$,

3. $d_{1} z A_{n}-B_{n}(z)=-1$.

We can, without loss of generality, assume $c_{0}=1$, and it then follows that

$$
\frac{A_{n}(z)}{B_{n}(z)}-\frac{b_{1}}{z}=\frac{-1}{d_{1} z B_{n}(z)}
$$

This commences with a term in $1 / z^{n+1}$ when expanded in powers of $1 / z$.

For the case where $b_{j}=0$ for $j>N>1$ and $b_{j} \neq 0$ for $j \leqslant N$ then the $M$ fraction can be obtained from the algorithm by setting $d_{i}^{(N-1)}=-n_{i}^{(N-1)}, i=2,3, \ldots$.

Example. The function

$$
f(z)=\left(1-e^{-z}\right) / z
$$

has the series expansion

$$
1-\frac{z}{2 !}+\frac{z^{2}}{3 !}-\frac{z^{3}}{4 !}+\cdots+\frac{(-)^{r} z^{r}}{(r+1) !}+\cdots,
$$

which is valid for all finite values of $z$. 
For $|z|$ large and $z$ in the right-hand half plane we see that $f(z)$ behaves like $1 / z$.

The continued fraction

$$
\frac{1}{1+z}-\frac{z / 2}{1+z / 2}-\frac{z / 3}{1+z / 3}-\cdots-\frac{z / r}{1+z / r}+\cdots
$$

corresponds to (7) and to the single term $1 / z$.

When $z$ is real the above example is a special case of the function

$$
F(p, x)=e^{-x p} \int_{0}^{x} e^{t^{p}} d t, \quad x \geqslant 0, p \geqslant 0
$$

and we see $F(1, x)=x f(x)$. Dijkstra [4] considered this function and gave the continued fraction expansion

$$
F(p, x)=\frac{a x}{a+t}-\frac{t}{a+1+t}-\frac{2 t}{a+2+t}-\cdots-\frac{r t}{a+r+t}-\cdots,
$$

where $t=x^{p}$ and $a=1 / p$, and this corresponds to the series expansions, in powers and inverse powers of $t$, of (9). The function (9) and the continued fraction (10) are generalizations of the special case $p=2$, namely Dawson's integral, considered by McCabe [1].

Dijkstra [4] provides a truncation error estimate for (10) and hence for (8), and also gives the number of terms of (8) that are required to estimate $f(x)$ to within ten significant figures for various values of $x$.

Finally, it is interesting to note that the denominators of the convergents of (8) are the partial sums of the exponential series while the numerators are the partial sums of the series expansion of $\left(e^{z}-1\right) / z$. That is, if we write $f(z)$ in the form $\left(\left(e^{z}-1\right) / z\right) / e^{z}$, then the successive numerator and denominator polynomials are the partial sums of the series expansions of the numerator and denominator, respectively.

Mathematical Institute

University of St. Andrews

St. Andrews, Fife, United Kingdom

1. J. H. McCABE, “A continued fraction expansion, with a truncation error estimate, for Dawson's integral," Math. Comp., v. 28, 1974, pp. 811-816. MR 51 \#7243.

2. J. H. MCCABE, "A formal extension of the Padé table to include two point Padé quotients," J. Inst. Math. Appl., v. 15, 1975, pp. 363-372. MR 52 \#2143.

3. J. H. MCCABE \& J. A. MURPHY, "Continued fractions which correspond to power series expansions at two points," J. Inst. Math. Appl., v. 17, 1976, pp. 233-247.

4. D. DIJKSTRA, “A continued fraction expansion for a generalization of Dawson's integral," Math. Comp., v. 31, 1977, pp. 503-510.

5. W. B. JONES, Multiple Point Padé Tables, Proc. Conf. Rational Approximation with Emphasis on Padé Approximants (Univ. of South Florida, December 1976). (To appear.) 